\title{
Poor modules with no proper poor direct summands
}

\author{
Rafail Alizade $^{\mathrm{a}}$, Engİn Büyükaşık ${ }^{\mathrm{b}}$, \\ Sergio R. López-Permouth ${ }^{\mathrm{c}, *}$, Liu Yang ${ }^{\mathrm{d}}$ \\ a Yaşar University, Department of Mathematics, 35100, Bornova, Izmir, Turkey \\ b Izmir Institute of Technology, Department of Mathematics, Gülbahçeköyü, 35430, \\ Urla, Izmir, Turkey \\ c Department of Mathematics, Ohio University, Athens, OH 45701, USA \\ d Jilin University, College of Mathematics, Jilin University, Changchun, Jilin, \\ 130012, China
}

\section{A R T I C L E I N F O}

\section{Article history:}

Received 17 August 2017

Available online 31 January 2018

Communicated by Louis Rowen

\section{$M S C$ :}

$13 \mathrm{C} 05$

$13 \mathrm{C} 11$

$13 \mathrm{C} 99$

20E34

20E99

\section{Keywords:}

Injective module

Poor module

Pauper module

\section{A B S T R A C T}

As a mean to provide intrinsic characterizations of poor modules, the notion of a pauper module is introduced. A module is a pauper if it is poor and has no proper poor direct summand. We show that not all rings have pauper modules and explore conditions for their existence. In addition, we ponder the role of paupers in the characterization of poor modules over those rings that do have them by considering two possible types of ubiquity: one according to which every poor module contains a pauper direct summand and a second one according to which every poor module contains a pauper as a pure submodule. The second condition holds for the ring of integers and is just as significant as the first one for Noetherian rings since, in that context, modules having poor pure submodules must themselves be poor.

It is shown that the existence of paupers is equivalent to the Noetherian condition for rings with no middle class. As indecomposable poor modules are pauper, we study rings with no indecomposable right middle class (i.e. the ring whose indecomposable right modules are pauper or injective). We show that semiartinian V-rings satisfy this property and also that a commutative Noetherian ring $R$ has no

\footnotetext{
* Corresponding author.

E-mail addresses: rafail.alizade@yasar.edu.tr(R. Alizade), enginbuyukasik@iyte.edu.tr (E. Büyükaşık), lopez@ohio.edu (S.R. López-Permouth), liuyang2000@jlu.edu.cn (L. Yang).
} 
indecomposable middle class if and only if $R$ is the direct product of finitely many fields and at most one ring of composition length 2. Structure theorems are also provided for rings without indecomposable middle class when the rings are Artinian serial or right Artinian.

Rings for which not having an indecomposable middle class suffices not to have a middle class include commutative Noetherian and Artinian serial rings. The structure of poor modules is completely determined over commutative hereditary Noetherian rings. Pauper Abelian groups with torsion-free rank one are fully characterized.

(c) 2018 Elsevier Inc. All rights reserved.

\section{Introduction}

While poor modules have been studied from the perspective of the injective profile of the ring, an intrinsic characterization of poor modules is also desirable. An immediate obstacle is that, since the injectivity domain of the direct sum of two modules is the intersection of the domains of injectivity of the summands, any module having a poor module as a direct summand will itself be poor. So, there might not be, in principle, anything interesting about the structure of certain summands and one must focus on those summands that are inherently poor. That leads us to the notion of a pauper. A module is a pauper (or a pauper module) if it is poor and no proper direct summand of it is poor.

Our interest in paupers is two-fold: we are interested in rings that have pauper modules and, most of all, in rings where the poverty of any module can be traced to having a pauper direct summand. Thus, for a ring $R$ and a class of right modules $\mathcal{C}$, we consider the properties

- $\mathcal{C}$ satisfies (E) if it contains paupers (existence), and

- $\mathcal{C}$ satisfies (U) for every poor module $P$ in $\mathcal{C}$ there exists a pauper module $M \in \mathcal{C}$ such that $M \subseteq \subseteq^{\oplus}$ (ubiquity).

When the class $\mathcal{C}$ is $\operatorname{Mod}-R$, we say that the $\operatorname{ring} R$ satisfies $(\mathrm{E})$ or $(\mathrm{U})$ when $\mathcal{C}$ does. While $(U)$ necessarily implies $(E)$, the converse may not be true. For instance, one can see in [2] that the ring of integers has paupers but it is not necessarily clear whether every poor module has a pauper direct summand.

Artinian serial rings satisfy (U) (Proposition 5.9). The class of torsion abelian groups and the class abelian groups with torsion-free rank one satisfy (U) (see [2, Corollary 3.2] and Corollary 6.7 respectively). As condition (U) seems to be difficult to attain, for a class of right modules $\mathcal{C}$ we consider a weaker property:

- $\mathcal{C}$ satisfies $\left(\mathrm{U}^{*}\right)$ if for every poor module $P$ in $\mathcal{C}$ there exists a pauper $M \in \mathcal{C}$ such that $M$ is a pure submodule of $P$. 
Condition ( $\left.\mathrm{U}^{*}\right)$ is just as significant as (U) for Noetherian rings in light of Theorem 4.1. Commutative hereditary Noetherian ring satisfy $\left(\mathrm{U}^{*}\right)$ (see, Corollary 4.9). Also, the class of modules with zero radical over a commutative semiartinian ring satisfies $\left(\mathrm{U}^{*}\right)$ (Corollary 5.4).

Notice that one rules out the less technical condition that every proper may simply contain a pauper as inconsequential: a module may contain paupers without being poor. In fact, a range of possibilities occur: Proposition 2.7 illustrates rings where modules that fail to contain a pauper must be injective yet Proposition 2.8 displays rings for which all modules contain paupers.

To every right module $M_{R}$ over an associative ring $R$ with unity there corresponds the class of modules with respect to which $M$ is injective. This class, its injectivity domain, ranges through all hereditary torsion classes in the interval from $S S M$ od- $R$ (the class of all semisimple right $R$-modules) to the class $\operatorname{Mod}-R$ (all right $R$-modules). The class of all injectivity domains is called the right injectivity profile (or right injective profile) of $R$ in [16]. For the sake of removing reference to specific modules, domains of injectivity are called portfolios and so the injective profile is the class of all portfolios. It was shown in [16] that the injective profile is always in a one-to-one correspondence with a set. The introduction of the so-called poor modules in [1] was the motivation for [16]. Poor modules are those whose injectivity domain is the class of semisimple modules.

In addition to providing several basic facts about poor modules (albeit not guaranteeing their existence for all rings, something that was only later accomplished in [9]) [1] proposed many directions for further inquiry and provided suitable terminology. Among other concepts, [1] introduces the idea, given a $\operatorname{ring} R$ and a class of right modules $\mathcal{A}$, that $R$ has no $\mathcal{A}$ middle class if the elements of $\mathcal{A}$ must all be either poor or injective. When $\mathcal{A}=\operatorname{Mod}-R$, one simply says that $R$ has no middle class. While semisimple Artinian rings are precisely those with singleton profile, the next simplest case to consider is therefore rings without middle class. The case when $\mathcal{A}=S S M$ od- $R$ has no middle class is considered in [6].

For any given class $\mathcal{A}$ of modules, two extreme situations are of interest. Following [1], when no module in $\mathcal{A}$ is poor, we say that the ring is an $\mathcal{A}$-utopia and, in the extreme opposite end of the spectrum, when every module in $\mathcal{A}$ is poor we say that the ring is $\mathcal{A}$-destitute. In [10], the authors study cyclic-destitute rings without a middle class.

Section 2 addresses the existence or absence of pauper modules in various families of rings. Semilocal rings are shown to have them while right semiartinian $V$-rings do not. Commutative rings with no middle class are Noetherian [6, Corollary 4.4]. In general, it is not known whether a ring with no middle class is right Noetherian. If a non Noetherian ring with no middle class exists, then such a ring must be a right semiartinian right $V$-ring by [9, Lemma 9]. Consequently, Proposition 2.7, shows that the existence of pauper modules is equivalent to the Noetherian condition for rings without middle class.

Clearly, an indecomposable poor module is pauper; however, the converse is far from true as paupers need not be indecomposable. In fact, no pauper over the integers can be indecomposable as follows: poor abelian groups have infinite uniform dimension and are 
not torsion-free (by [2]), but then, since Kulikov's theorem implies that indecomposable abelian groups that are not torsion-free must be uniform (c.g. 5.2.10 in page 98 of [21]), no pauper abelian group is indecomposable. It makes sense at this junction to consider those rings that lack middle class for indecomposable modules; that is the subject of Section 3. In that section, we show that semiartinian V-rings satisfy this property and consider cases when the absence of an indecomposable middle class suffices to guarantee that the ring has no middle class. We also show that a commutative Noetherian ring $R$ has no indecomposable middle class if and only if $R$ is the direct product of finitely many fields and at most one ring of composition length 2 and provide structure theorems for rings without indecomposable middle class when the rings involved are Artinian serial or right Artinian.

Condition (E) highlights an interesting relationship between the semiartinian and $V$-ring conditions. Under the semiartinian condition, a ring that satisfies (E) cannot be $V$-ring (Proposition 2.5). We show that the converse is true when the ring is commutative (Proposition 5.2).

When $C$ is a complete set of representatives of non-injective simple $R$-modules, we will consistently use the notation $S=\oplus_{U \in C} U$ and refer to this module as the irredundant complete sum of noninjective simples. The module $S$ pops up in various places in the paper.

Unless otherwise noted, all modules are right and unital. A ring $R$ is a right $V$-ring if every simple right module is injective (see [18]). A ring $R$ is a right PCI ring if every cyclic right module not isomorphic to $R$ is injective. For all other standard module theoretic terminology, the reader is referred to [5], [11], or [15]. When dealing with notions of purity, a good reference is [20].

\section{Pauper modules: poor modules with no proper poor direct summands}

While considering the existence of pauper modules, Proposition 2.1 shows that the class of finite uniform dimensional modules satisfies (U). Therefore, if the $\operatorname{ring} R$ is not a finite-uniform-dimension-utopia (i.e. if there exist poor modules with finite uniform dimension) then the $R$ satisfies (E). The proposition finds an immediate application to show that semilocal rings satisfy (E) (Proposition 2.2).

Proposition 2.1. Over arbitrary rings, finite uniform dimensional poor modules have pauper direct summands.

Proof. Suppose $M$ is a finite uniform dimensional poor module over a $\operatorname{ring} R$. If $M$ is not a pauper, then there are nonzero submodules $A_{1}$ and $B_{1}$ of $M$ such that $M=A_{1} \oplus B_{1}$ and $A_{1}$ is poor. If $A_{1}$ is pauper, then we are done. Otherwise, there are nonzero submodules $A_{2}$ and $B_{2}$ of $A_{1}$ with $A_{2}$ is poor such that $A_{1}=A_{2} \oplus B_{2}$. Since $M$ has finite uniform dimension and $M>A_{1}>A_{2}$ is a chain of direct summand of $M$, by the same procedure we shall obtain a pauper submodule of $M$ after finitely many steps. 
We show next that semilocal rings satisfy $(\mathrm{E})$.

Proposition 2.2. Let $R$ be a semilocal ring. Then the right $R$-module $\frac{R}{J(R)}$ has a pauper direct summand.

Proof. By [7, Proposition 5.1] the $R$-module $\frac{R}{J(R)}$ is poor. On the other hand, $\frac{R}{J(R)}$ has finite uniform dimension. Then the proof follows by Proposition 2.1.

Clearly, an indecomposable poor module is pauper and, as shown above, finite uniform dimensional poor modules have pauper direct summands. The following examples should dispell any expectations that being pauper is close to being indecomposable or finite dimensional.

Example 2.3. (1) The abelian group $\oplus_{p} \mathbb{Z}_{p}$ is pauper by [2, Theorem 3.1].

(2) Let $G=\prod_{p} \mathbb{Z}_{p}$. Then, again, $G$ is poor by [2, Theorem 3.1]. Let us show that, $G$ is pauper. Suppose $G=H \oplus K$ for some poor submodule $H$ of $G$. Then $\oplus_{p} \mathbb{Z}_{p}$ is contained in $H$ by $\left[2\right.$, Theorem 3.1]. Then $K \cap\left(\oplus_{p} \mathbb{Z}_{p}\right)=0$. Note that $\frac{G}{\left(\oplus_{p} \mathbb{Z}_{p}\right)} \cong \mathbb{Q}^{(I)}$ and $\operatorname{Rad}(G)=0$. Since $K \cap\left(\oplus_{p} \mathbb{Z}_{p}\right)=0$ and $\frac{G}{\left(\oplus_{p} \mathbb{Z}_{p}\right)} \cong K \oplus \frac{H}{\left(\oplus_{p} \mathbb{Z}_{p}\right)}, K$ is divisible. Then $\operatorname{Rad}(K)=K$. This implies that $K=0$, because $\operatorname{Rad}(K) \subseteq \operatorname{Rad}(G)=0$. Therefore $G$ is pauper.

By [9], poor modules exist over any ring. At this point, the first question that comes to mind is whether pauper modules exists over any ring. To show that pauper modules do not exist in general, we need the following lemma.

Lemma 2.4. Let $R$ be any ring and $E$ an injective right $R$-module. Let $N$ be a right $R$-module. Then $E \oplus N$ is poor if and only if $N$ is poor.

Proof. Let $B$ a right $R$-module. Since $E$ is injective, $E \oplus N$ is $B$-injective if and only if $N$ is $B$-injective (see, [19, Theorem 1.7]). Now the proof is clear.

It is clear that a ring $R$ is semisimple Artinian if and only if the trivial module 0 is pauper. In fact, semisimple Artinian rings are a trivial example of rings satisfying condition (U).

Proposition 2.5. Let $R$ be a (nonsemisimple) right semiartinian right $V$-ring. Then $R$ has no pauper right $R$-module.

Proof. By [9], every ring has a poor module. Let $M$ be a poor right $R$-module. Then $\operatorname{Soc}(M) \neq 0$ by the semiartinian condition. Let $U$ be a simple submodule of $M$. Then $U$ is injective by the hypothesis, and so $M=U \oplus N$ for some submodule $N$ of $M$. Since $U$ is injective, $N$ is poor by Lemma 2.4. This show that any poor right $R$-module contains a proper poor direct summand. Hence $R$ has no pauper right module. 
Corollary 2.6. Let $R$ be a (non semisimple) right $V$-ring. Suppose $R$ has a pauper right module $M$. Then $\operatorname{Soc}(M)=0$. Moreover, every semiartinian right module is semisimple.

Proof. Let $M$ be a pauper right module. Then, arguing as in the proof of Proposition 2.5, $\operatorname{Soc}(M)=0$. For any Semiartinian right module $N$ and $K \leq N$, it is clear that $\operatorname{Hom}(K, M)=0$. Thus $M$ is $N$-injective. Since $M$ is poor, $N$ must be semisimple.

In [1], a ring $R$ is said to have no right middle class if each right $R$-module is either injective or poor. For characterizations of the rings with no middle class we refer to [9] and [6].

It is not known in general whether a ring without right middle class is right Noetherian; we show next that, for those rings, that condition is indeed equivalent to the existence of paupers.

Proposition 2.7. Let $R$ be a ring with no right middle class. The following statements are equivalent.

(1) $R$ is right Noetherian.

(2) $R$ has a pauper right module.

(3) Every noninjective right module has a pauper submodule.

(4) Every noninjective right $R$-module has a cyclic pauper submodule.

Proof. (4) $\Rightarrow(3) \Rightarrow(2)$ are clear. (2) $\Rightarrow(1)$ Suppose $R$ is not right Noetherian. Then $R$ is a right semiartinian right $V$-ring by [9, Lemma 5 and Lemma 9]. Then $R$ has no pauper module by Proposition 2.5. This contradicts (2). Hence $R$ must be right Noetherian.

(1) $\Rightarrow$ (4) Let $M$ be a noninjective right $R$-module. By (1) we can assume that $M$ has no nonzero injective submodule. Let $N$ be a nonzero cyclic submodule of $M$. Then $N$ is poor by the hypothesis. Clearly $N$ has finite uniform dimension. So, by Proposition 2.1, $N$ has a pauper direct summand (necessarily cyclic). This proves (4).

Proposition 2.8. Let $R$ be a right $V$-ring with no right middle class. The following are equivalent.

(1) $R$ is semisimple Artinian.

(2) $R$ has a pauper right module.

(3) Every right module contain a pauper submodule.

Proof. Proposition 2.7 and [9, Lemma 9].

Summing up, we have the following by [9, Theorem 2], Proposition 2.7 and Proposition 2.8 . 
Corollary 2.9. Let $R$ be a ring with no right middle class. Then $R$ has a pauper right module if and only if $R=S \times T$, where $S$ is semisimple and $T$ is Noetherian.

\section{Rings with no indecomposable right middle class}

Let $R$ be a ring with no right middle class. Then a right module is pauper if and only if it is noninjective and indecomposable. Thus at this point it is natural to consider the rings whose indecomposable right modules are poor (thus pauper) or injective. To be consistent with the terminology in [1], we say that $R$ has no indecomposable right middle class if every indecomposable right module is poor or injective.

Any ring with no right middle class is a ring with no indecomposable right middle class. The converse holds for commutative Noetherian rings and for Artinian serial rings (respectively, Corollary 3.6 and Theorem 3.7 below). In general, however, we do not know yet whether the two conditions are equivalent. The following Proposition might be a step in that direction.

Proposition 3.1. A right semiartinian right $V$-ring is a ring with no indecomposable middle class.

Proof. Under the hypotheses, indecomposable modules are, in fact, (simple and) injective.

Proposition 3.2. Let $R$ be a ring with no indecomposable middle class and $I$ an ideal of $R$. Then the ring $\frac{R}{I}$ has no indecomposable middle class.

Proof. Let $I$ be an ideal of $R$ and $M$ be an indecomposable right $\frac{R}{I}$-module. Then $M$ is also an indecomposable $R$-module. Since $R$ has no indecomposable middle class, $M$ is an injective or a poor $R$-module. Then $M$ is injective or poor as an $\frac{R}{I}$-module.

The following two lemmas might be well-known but we could not find an easy reference so we decided to include them here for completeness and the convenience of the reader.

Lemma 3.3. Let $R$ be a commutative ring and $A$ and $B$ be two $R$-modules of composition length two and with isomorphic simple socle $V$. Then $\frac{A}{V} \cong \frac{B}{V} \cong V$ and, in fact, $A \cong B$ and, moreover, $A$ is A-injective.

Proof. By the hypothesis $A$ and $B$ are not semisimple. Suppose $\frac{A}{V} \cong U$ for some simple module $U$ which is not isomorphic to $V$. Let $P=\operatorname{ann}_{R}(V)$ and $Q=\operatorname{ann}_{R}(U)$. Then $P Q A=0$, and so $A$ is an $\frac{R}{P Q}$-module. Since $\frac{R}{P Q} \cong \frac{R}{P} \oplus \frac{R}{Q}$ is semisimple, $A$ is a semisimple $\frac{R}{P Q}$-module, and so a semisimple $R$-module. Contradiction. Hence $\frac{A}{V} \cong V$. As $V \unlhd A$ and $V \unlhd B$, we have $\operatorname{Rad} A=V \ll A$ and $\operatorname{Rad} B=V \ll B$. These implies $A=R a$ and $B=R b$ are cyclic. Now it is easy to see that $\operatorname{ann}_{R}(A)=a n n_{R}(B)$, and so $R a \cong \frac{R}{I} \cong R b$ for some ideal $I$ of $R$. Hence $A \cong B$. 
To prove the second part, we shall show that $A$ is $A$-injective. For this it is enough to prove that if $X$ is a submodule of $A$ with $X \cong V$, then any homomorphism $f: X \rightarrow A$ can be extended to a homomorphism $g: A \rightarrow A$. Without loss of generality we may assume that $X=V$ and $f$ is nonzero. Clearly, $A$ and $V$ are cyclic modules. Suppose that $A=a R$ and $V=x R$. Then $f(x)=x s$ for some $s \in R$, because $f(V) \subseteq V$. Define $g: A \rightarrow A$ by $g(b)=b s$ for each $b \in A$. Then $g(x)=x s=g(x)$, and so $g$ extends $f$. Therefore $A$ is $A$-injective.

Lemma 3.4. For a simple module $V$ over a commutative Noetherian ring, the properties of injectivity, flatness and projectivity are equivalent.

Proof. Over a commutative ring a simple module $V$ is injective if and only if it is flat by [22, Lemma 2.6.]. In addition, if the ring is Noetherian, then $V$ is finitely presented. By [15, Theorem 4.30] finitely presented flat modules are projective. Therefore for a simple module $V$ over a commutative Noetherian ring being injective, flat and projective are equivalent conditions.

Theorem 3.5. Let $R$ be a commutative Noetherian ring. Then $R$ has no indecomposable middle class if and only if $R=S \times T$, where $S$ is semisimple Artinian and $T$ is either zero or a local ring whose maximal ideal is minimal. In other words, $R$ is the direct product of finitely many fields and at most one ring of composition length 2.

Proof. Suppose $R$ has no indecomposable middle class. Let $S$ be the sum of the injective minimal ideals of $R$. Then $S$ is injective by the Noetherian assumption, and so $R=S \oplus T$ for some ideal $T$ of $R$. Suppose $T \neq 0$. Then $T$ is not semisimple.

First let us prove that $T$ is a local ring. Assume $T$ is not local and let $P$ and $Q$ be two distinct maximal ideals of $T$. By Lemma 3.4, and the fact that $S \cap T=0$, $T$ has no injective simple factor. Thus $U=\frac{T}{P}$ and $U^{\prime}=\frac{T}{Q}$ are noninjective simple modules. Then $U$ and $U^{\prime}$ are poor by the hypothesis. On the other hand, $E(U)$ is Artinian by [17, Proposition 3], and so $\operatorname{Soc}\left(\frac{E(U)}{U}\right) \neq 0$. Then there is a submodule $A$ of $E(U)$ such that $\frac{A}{U}$ is simple. Then, as $\operatorname{Hom}\left(U, U^{\prime}\right)=0, U^{\prime}$ is $A$-injective. This contradicts the fact that $U^{\prime}$ is poor. Therefore $T$ must be local.

Let $U$ be the unique simple $T$-module and $A$ as above. Then $A$ is $A$-injective by Lemma 3.3. But $A$ is also indecomposable, so $A$ must be injective by the hypothesis again. Thus we get $A=E(U)$. Let us prove that $T$ is Artinian by showing that it is semiartinian. Suppose $\operatorname{Soc}(M)=0$ for some cyclic $T$-module $M$. Without loss of generality we may assume that $M$ is noninjective (if it were injective, take a cyclic noninjective submodule of $M$ ) and, by the Noetherian condition, indecomposable. Then $M$ is poor by the hypothesis. On the other hand, $\operatorname{Hom}(A, M)=0$ because $\operatorname{Soc}(M)=0$. This means that $M$ is $A$-injective. But $M$ is poor, a contradiction. Therefore $T$ is Artinian.

Now $\operatorname{Soc}(T)$ is finitely generated, and so $\operatorname{Soc}(T) \cong U^{n}$ for some positive integer $n$. Since $\operatorname{Soc}(T)$ is essential in $T$, $\operatorname{Soc}(T) \leq T \leq A^{n}$. Thus $\frac{T}{\operatorname{Soc}(T)}$ can be embedded in 
the semisimple module $\left(\frac{A}{U}\right)^{n}$, and so $\frac{T}{\operatorname{Soc}(T)}$ is semisimple. Since $T$ is local and $\frac{A}{U} \cong U$, we must have $\frac{T}{\operatorname{Soc}(T)} \cong U$. Then $J(T)=\operatorname{Soc}(T)$, and so $(\operatorname{Soc}(T))^{2}=0$. Since $A$ is nonsemisimple and has composition length two, it is cyclic, i.e. $A=a T$ for some $a \in A$. Let us prove that $\operatorname{ann}_{R}(a)=0$. Suppose there is a nonzero $r \in T$ such that $r a=0$. Clearly $r$ is not a unit, and so $r \in \operatorname{Soc}(T)$. Since $T$ is local and the socle of $T$ is homogeneous $r T=U^{n}$ for some $n \in N$. Thus $U a=0$, and $\operatorname{so} \operatorname{Soc}(T) a=0$. This implies that $A$ is a $\frac{T}{\operatorname{Soc}(T)}$-module. Since $\frac{T}{\operatorname{Soc}(T)}$ is simple, we get that $A$ is semisimple. Contradiction. Therefore $\operatorname{ann}_{R}(a)=0$, and so the homomorphism $f: T \rightarrow A$ defined as $f(t)=a t$ is an isomorphism. This proves the necessity.

To prove the sufficiency, let $M$ be a noninjective and indecomposable module. By the hypothesis $M=M_{1} \oplus M_{2}$, where $M_{1}$ is an $S$-module and $M_{2}$ is a $T$-module. Since $M$ is indecomposable and noninjective, $M_{1}=0$. Thus we must have, $M=M_{2} \cong U$, where $U$ is the unique simple $T$-module. Then $M$ is poor by Proposition 2.2 .

In particular, the previous theorem shows that the absence of an indecomposable middle class assures that the ring itself has no middle class when the ring is Noetherian and commutative, a result we state for the record in the following corollary.

Corollary 3.6. Let $R$ be a commutative Noetherian ring. Then $R$ has no middle class if and only if $R$ has no indecomposable middle class.

Proof. By Theorem 3.5 and [6, Theorem 4.3].

The coincidence of lacking an indecomposable middle class with not having one at all also holds for Artinian serial rings, as we see next.

Theorem 3.7. Let $R$ be an Artinian serial ring. The following are equivalent.

(1) $R$ has no indecomposable middle class;

(2) $R$ has no right middle class;

(3) $R=S \times T$, where $S$ is semisimple Artinian and $T$ is a ring with $J(T)^{2}=0$.

Proof. (1) $\Rightarrow(2)$ Let $M$ be a noninjective right module. Then $M=\oplus_{i \in I} U_{i}$, where $U_{i}$ is uniserial for each $i \in I$ by [11, Theorem 5.6]. Since $M$ is noninjective, $U_{j}$ is noninjective for some $j \in I$. Then $U_{j}$ is poor by (1), and so $M$ is poor.

$(2) \Rightarrow(1)$ is clear.

$(2) \Leftrightarrow(3)$ By $[6$, Theorem 3].

Theorem 3.8. Let $R$ be a right Artinian ring. Suppose $R$ has no indecomposable middle class. Then $R=S \times T$, where $S$ is semisimple Artinian, $\operatorname{Soc}\left(T_{T}\right)$ is homogeneous, $J(T)=\operatorname{Soc}\left(T_{T}\right)$ and $J(T)^{2}=0$. 
Proof. Suppose $R$ has no indecomposable middle class. Then $R=S \times T$, where $S$ is semisimple Artinian and $T$ has a unique noninjective simple right module and $\operatorname{Soc}\left(T_{T}\right)$ is homogeneous by [7, Proposition 5.7]. Let $U$ be the unique noninjective simple right $T$-module, and $E(U)$ be its injective hull. Note that no simple right submodule of $T$ is a direct summand of $T$, again by the proof of [7, Proposition 5.7]. Thus $\operatorname{Soc}\left(T_{T}\right) \leq J(T)$. By the right Artinian condition, $\operatorname{Soc}\left(\frac{E(U)}{U}\right)=\frac{A}{U} \neq 0$. Then $A$ is quasi-injective by [8, Lemma 4]. Since $A$ is indecomposable and $R$ has no indecomposable middle class, $A$ is injective. Thus we have, $A=E(U)$ and $\frac{E(U)}{U}$ is semisimple. Now $\operatorname{Soc}\left(T_{T}\right)$ is finitely generated by the Artinian condition, $\operatorname{so} \operatorname{Soc}\left(T_{T}\right) \cong U^{n}$ for some positive integer $n$. Then $\frac{T}{\operatorname{Soc}\left(T_{T}\right)}$ can be embedded in the semisimple module $\left(\frac{E(U)}{U}\right)^{n}$. Then $\frac{T}{\operatorname{Soc}\left(T_{T}\right)}$ is semisimple, and so $J(T) \leq \operatorname{Soc}\left(T_{T}\right)$. Thus, we have $J(T)=\operatorname{Soc}\left(T_{T}\right)$ and $J(T)^{2}=0$. This completes the proof.

\section{Poor and pauper modules over Noetherian rings}

The structure of poor $\mathbb{Z}$-modules was recently described in [2]. In this section, we study poor and pauper modules over Noetherian rings. Poor modules are fully characterized over commutative hereditary Noetherian rings.

The following theorem is useful for investigation of the structure of poor modules over Noetherian rings. It shows that the familiar fact that a module having a poor direct summand must itself be poor may be extended, over right Noetherian rings, to modules having poor pure submodules. Note that the dual result for modules over Artinian rings is proved in [4, Theorem 3.2].

Theorem 4.1. Let $R$ be a right Noetherian ring and $M$ be a right $R$-module. If $L$ is a pure submodule of $M$ and $L$ is poor, then $M$ is poor.

Proof. It is sufficient to show that if $M$ is $A$-injective for some cyclic right $R$-module $A$, then $L$ is also $A$-injective. Let $B$ be a submodule of $A$ and $f: B \rightarrow L$ be a homomorphism. Since $M$ is $A$-injective, there is a homomorphism $g: A \rightarrow M$ such that $\left.g\right|_{B}=f$. So we have the following diagram with exact rows.

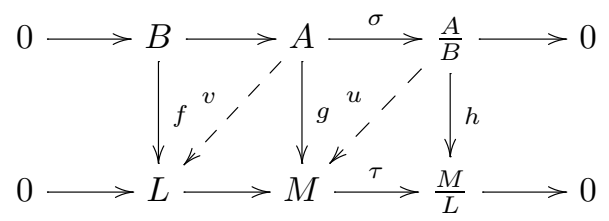

Since $R$ is Noetherian and $A$ is cyclic, $B$ is finitely generated. So $\frac{A}{B}$ is finitely presented. Since the bottom row is pure exact there is a homomorphism $u: \frac{A}{B} \rightarrow M$ such that $\tau u=h$ (see [20, Lemma 3.70]). Then, by [13, Lemma 8.4], there is a homomorphism $v: A \rightarrow L$ such that $\left.v\right|_{B}=f$. That is $L$ is $A$-injective. This completes the proof. 
The Noetherian condition in Theorem 4.1 is not superfluous. Let $R$ be a nonsemisimple right semiartinian right $V$-ring. Then it is easy to see that each uniform right module is simple. This fact together with [9, Proposition 2] implies that the direct sum of non-isomorphic simple right modules $\oplus_{S_{i} \in A} S_{i}$ is poor. On the other hand $\oplus_{S_{i} \in A} S_{i}$ is a pure submodule of $\prod_{S_{i} \in A} S_{i}$, while $\prod_{S_{i} \in A} S_{i}$ is not poor as it is injective.

Proposition 4.2. Let $R$ be a commutative Noetherian ring and $C$ be a complete set of representatives of noninjective simple $R$-modules. Then $S=\oplus_{S_{i} \in C} S_{i}$ is pauper. Moreover, for any poor $R$-module $M$, the singular submodule $Z(M)$ of $M$ contains a copy of $S$.

Proof. First we shall prove that $S$ is poor. Suppose that $S$ is $A$-injective for a cyclic module $A$. Then $A \cong \frac{R}{I}$, for some ideal $I$ of $R$. Let $S^{\prime}$ be the direct sum of a complete set of representatives of noninjective simple $\frac{R}{I}$-modules. Then, clearly, $S^{\prime}$ is isomorphic to a direct summand of $S$. As $S$ is $A$-injective, so is $S^{\prime}$, and therefore, $S^{\prime}$ is $\frac{R}{I}$-injective, which is a contradiction unless $S^{\prime}=0$. But if $\frac{R}{I}$ has no noninjective simples then $\frac{R}{I}$ is a commutative Noetherian V-ring and therefore it is semisimple. So $A$ is a semisimple $R$-module, proving that $S$ is poor, as claimed.

To see that $S$ is pauper, let $N$ be proper direct summand of $S$. Then there is a noninjective simple $R$-module $T$ such that $\operatorname{Hom}(T, N)=0$. By [17, Proposition 3], $E(T)$ is Artinian. Since $T$ is noninjective, there is a submodule $A$ of $E(T)$ with composition length 2. Clearly $A$ is not semisimple and $N$ is $A$-injective. Hence $N$ is not poor, and so $S$ is pauper.

To prove the last part, let $M$ be a poor module and $V$ be a noninjective simple module. Then $\operatorname{Hom}(V, M) \neq 0$ by the previous paragraph. By Lemma $3.4 V$ is not projective. Hence $V \subseteq Z(M)$, and this implies $S \subseteq Z(M)$. This completes the proof.

Corollary 4.3. Let $R$ be a commutative Noetherian ring. Then any module $N$ such that $\oplus_{S_{i} \in C} S_{i} \subseteq N \subseteq \prod_{S_{i} \in C} S_{i}$ is poor.

Proof. Let $N$ be such that $\oplus_{S_{i} \in C} S_{i} \subseteq N \subseteq \prod_{S_{i} \in C} S_{i}$. Since $\oplus_{S_{i} \in C} S_{i}$ is a pure submodule of $\prod_{S_{i} \in C} S_{i}, \oplus_{S_{i} \in C} S_{i}$ is a pure submodule of $N$. Hence $N$ is poor by Theorem 4.1 and Proposition 4.2.

Lemma 4.4. Let $R$ be a commutative Noetherian ring and $A=\left\{S_{i}\right\}_{i \in I}$ be a complete set of representatives of non-isomorphic simple $R$-modules. Then the module $M=\frac{\prod_{i \in I} S_{i}}{\oplus_{i \in I} S_{i}}$ has no maximal submodules (i.e. $\operatorname{Rad}(M)=M$ ).

Proof. Let $P$ be a maximal ideal of $R$. Then $P=\operatorname{ann}_{R}\left(S_{i}\right)$ for some $S_{i} \in A$ and $P S_{j}=S_{j}$ for all $S_{j} \in A$ with $S_{j} ¥ S_{i}$. Therefore $P\left(\prod_{i \in I} S_{i}\right)=\prod_{j \neq i} S_{j}$, and $P M=$ $\frac{\prod_{j \neq i} S_{j}+\left(\oplus_{i \in I} S_{i}\right)}{\oplus_{i \in I} S_{i}}=M$. Hence we get $\operatorname{Rad}(M)=\cap Q M=M$, where $Q$ ranges over the set of maximal ideals of $R$, i.e. $M$ has no maximal submodules.

The following proposition provides some examples of pauper modules. 
Proposition 4.5. Let $R$ be commutative hereditary Noetherian ring and $\left\{S_{i}\right\}_{i \in I}$ be a complete set of representatives of non-isomorphic simple $R$-modules. Than any module $N$ such that $\oplus_{i \in I} S_{i} \subseteq N \subseteq \prod_{i \in I} S_{i}$ and $\operatorname{Rad}\left(\frac{N}{\left(\oplus_{i \in I} S_{i}\right)}\right)=\frac{N}{\left(\oplus_{i \in I} S_{i}\right)}$ is pauper. In particular, $\prod_{i \in I} S_{i}$ is pauper.

Proof. First note that $\operatorname{Rad}\left(\prod_{i \in I} S_{i}\right)=0$. Suppose that $N=A \oplus B$ with $A$ is poor. Then $\oplus_{i \in I} S_{i} \subseteq A$ by Proposition 4.2. Let $\pi_{B}: N \rightarrow B$ be the natural projection. Then $\oplus_{i \in I} S_{i} \subseteq \operatorname{Ker}\left(\pi_{B}\right)$, and so there is an epimorphism $\bar{\pi}_{B}: \frac{N}{\left(\oplus_{i \in I} S_{i}\right)} \rightarrow B$. Now, $\operatorname{Im}\left(\bar{\pi}_{B}\right) \subseteq \operatorname{Rad}(B)=0$, by the hypothesis. Therefore $B=0$, and so $N$ is pauper.

The remaining conclusions follow from Lemma 4.4.

Proposition 4.6. Let $M$ be an R-module. Suppose that for every noninjective simple module $V, M$ has a direct summand isomorphic to $V$. Then $M$ has a pure submodule isomorphic to $S$, where $S$ is the irredundant complete direct sum of noninjective simple R-modules.

Proof. Let $U$ and $V$ be nonisomorphic, noninjective simple $R$-modules, that are direct summands of $M$. Then $M=U \oplus N$ for some $N \subseteq M$. Since $\pi_{U}(V)=0$ where $\pi_{U}$ : $M \rightarrow U$ is the projection on $U$ with $\operatorname{Ker}\left(\pi_{U}\right)=N, V \subseteq N$. But $V$ is a direct summand of $M$, so it is a direct summand of $N$ by Modular Law. Then $M=U \oplus V \oplus K$ for some $K \subseteq M$. The same can be proved by induction for any finite set of nonisomorphic simple submodules of $M$. Now if $\left\{U_{i}\right\}_{i \in I}$ is a complete set of representatives of the noninjective simple $R$-modules with each $U_{i}$ is a direct summand of $M$, then for every finite subset $F \subseteq I, N_{F}=\oplus_{i \in F} U_{i}$ is a direct summand and therefore a pure submodule of $M$. Since direct limit of pure submodules is pure, $S=\oplus_{i \in I} U_{i}=\lim _{\longrightarrow} N_{F}$ is a pure submodule of $M$. This completes the proof.

Lemma 4.7. Let $R$ be a commutative hereditary Noetherian ring. Let $M$ be an $R$-module and $V$ a simple submodule of $M$. The following are equivalent.

(1) $V$ is closed in $M$.

(2) $Q V=V \cap Q M$ for each maximal ideal $Q$ of $R$.

(3) $V$ is a direct summand of $M$.

Proof. $(1) \Leftrightarrow(2)$ By [3, Theorem 3.3].

$(2) \Rightarrow(3)$ Let $P=\operatorname{ann}(V)$. Then $0=P V=V \cap P M$ by (2), and so $V \nsubseteq P M$. Since $M / P M$ is semisimple, $P M$ is an intersection of maximal submodules of $M$. Therefore there is a maximal submodule, say $K$, of $M$ such that $V+K=M$. As $V$ is simple, we have $M=V \oplus K$. This proves (3).

$(3) \Rightarrow(1)$ is clear. 
Theorem 4.8. Let $R$ be a commutative hereditary Noetherian ring. An $R$-module $M$ is poor if and only if, for every noninjective simple $V, M$ has a direct summand isomorphic to $V$.

Proof. To prove the necessity, suppose that there is a noninjective simple $R$-module $V$ such that $M$ has no direct summand isomorphic to $V$. Since $V$ is noninjective, there is an $R$-module of length two such that $V \unlhd A$ and $\frac{A}{V} \cong V$. There are two cases.

Case I. $\operatorname{Hom}(V, M)=0$. Since $0<V<A$ is a composition series for $A$ with $\frac{A}{V} \cong V$, for every submodule $X$ of $A \operatorname{Hom}(X, M)=0$. Hence $M$ is $A$-injective, and so $M$ is not poor. Contradiction.

Case II. $\operatorname{Hom}(V, M) \neq 0$. Let $X$ be a nontrivial submodule of $A$ and $f: X \rightarrow M$ be any nonzero homomorphism. Without loss of generality we can assume that $X=V$. If $f(V)$ is a closed submodule of $M$, then $f(V)$ is a direct summand of $M$ by Lemma 4.7. That is, $M$ has a direct summand isomorphic to $V$. Contradiction. So $f(V)$ is not closed in $M$. Then there is a submodule $Y$ of $M$ such that $V \unlhd Y$. Then $Y / V$ is singular. So that there is a submodule $B$ of $Y$ of length two with $f(V) \unlhd B$. Then $A \cong B$ and $B$ is $A$-injective by Lemma 3.3. Hence $M$ is $A$-injective. Contradiction. This proves the necessity.

To prove the sufficiency suppose that for every noninjective simple module $V$, $M$ has a direct summand isomorphic to $V$. Then by Proposition 4.6 $M$ has a pure submodule $N$ isomorphic to $S$, the irredundant complete sum of noninjective simple $R$-modules. $N$ is poor by Theorem 4.2. Therefore $M$ is poor by Theorem 4.1.

The structure of poor abelian groups was characterized in [2]. Namely, an abelian group $G$ is poor if and only if the torsion part $T(G)$ of $G$ has a direct summand isomorphic to $\oplus_{p} \mathbb{Z}_{p}$, where $p$ ranges over the prime integers. The following theorem is a generalization of $\left[2\right.$, Theorem 3.1]; it shows that commutative hereditary Noetherian rings satisfy $\left(\mathrm{U}^{*}\right)$.

Theorem 4.9. A commutative hereditary Noetherian ring $R$ satisfies ( $\left.U^{*}\right)$. In fact, for every right $R$-module $M$, the following statements are equivalent.

(1) $M$ is poor.

(2) $Z(M)$ is poor.

(3) For every noninjective simple module $V$, $M$ has a direct summand isomorphic to $V$.

(4) $M$ has a pure submodule isomorphic to $S$, where $S$ is the sum of nonisomorphic and noninjective simple $R$-modules.

Proof. (1) $\Rightarrow(2)$ Suppose $M$ is poor and let $V$ be a noninjective simple $R$-module. Then $M$ has a direct summand, say $U$, isomorphic to $V$ by Theorem 4.8. Then $U$ is contained in $Z(M)$ by Theorem 4.2. Therefore $U$ is a direct summand of $Z(M)$, and so $Z(M)$ is poor by Theorem 4.8. 
$(2) \Rightarrow(1)$ Since $R$ is hereditary, $\frac{M}{Z(M)}$ is flat by [14, Proposition 2.3]. Then $Z(M)$ is a pure submodule of $M$, and so $M$ is poor by Theorem 4.1.

$(1) \Leftrightarrow(3)$ By Theorem 4.8.

$(3) \Rightarrow(4)$ By Proposition 4.6.

$(4) \Rightarrow(1)$ By Theorem 4.1 and Theorem 4.2.

\section{Poor and pauper modules over semiartinian rings}

In this section, we shall prove that $S$, the irredundant complete sum of noninjective simple modules, is pauper over commutative Semiartinian rings. Moreover, we prove that any poor module contains a copy of $S$.

Lemma 5.1. [7, Lemma 5.9] Let $R$ be a commutative ring and $V$ be a simple $R$-module. If $V$ is $B$-injective for some $R$-module $B$, then $V^{(I)}$ is $B$-injective for every index set $I$.

Proposition 5.2. Let $R$ be a commutative semiartinian ring. Then the irredundant complete sum of noninjective simple right $R$-modules is pauper. Moreover any poor module contains an isomorphic copy of each noninjective simple module.

Proof. Let $S$ be the irredundant complete direct sum of noninjective simple right $R$-modules. Suppose $S$ is $M$-injective for some cyclic right $R$-module $M$. Since $R$ is semiartinian, $\operatorname{Soc}(M) \unlhd M$. Assume $M$ is not semisimple. First, we claim that $\operatorname{Soc}(M)$ has finite length. Suppose the contrary. Then $M$ contains an infinite direct summand of $S$, say $N$ or contains a direct summand isomorphic to $V^{(I)}$, where $V$ is a noninjective simple module and $I$ an infinite index set. In the former case, $M$ is $N$-injective, so the inclusion $N \rightarrow M$ splits, a contradiction. In the latter case, since $V$ is $M$-injective, $V^{(I)}$ is $M$ injective by Lemma 5.1, and so the inclusion $V^{(I)} \rightarrow M$ splits, a contradiction. Therefore $\operatorname{Soc}(M)$ has finite length, and so $\operatorname{Soc}(M)$ is $M$-injective. Which implies that $\operatorname{Soc}(M)$ is a direct summand of $M$, a contradiction. Hence $M$ is semisimple, and so $S$ is poor.

Similar arguments of the proof of Theorem 4.2 shows that $M$ is pauper, and any poor module contains a copy of $S$.

Note that Proposition 5.2 is not true over noncommutative semiartinian rings (see, Proposition 2.5).

Proposition 5.3. Let $R$ be a commutative semiartinian ring and $A$ be a right $R$-module with $\operatorname{Rad} A=0$. The following are equivalent.

(1) A is poor.

(2) A has a pure submodule isomorphic to $S$, where $S$ is the direct sum of non injective simple modules. 
(3) For every noninjective simple right $R$-module $V, A$ has direct summand isomorphic to $V$.

Proof. $(1) \Rightarrow(2)$ By Proposition 5.2 and Proposition 4.6.

$(2) \Rightarrow(1)$ Similar to the proof of the first part of Proposition 5.2.

$(2) \Rightarrow(3)$ Let $V$ be non injective simple module. Since $\operatorname{Rad} A=0$, there is a maximal submodule $K$ of $A$ such that $V+K=A$. As $V$ is simple, $V \cap K=0$, so $A=V \oplus K$.

$(3) \Rightarrow(2)$ is clear.

Corollary 5.4. Let $R$ be a commutative semiartinian ring. The class of $R$-modules with zero radical satisfy $\left(U^{*}\right)$.

Corollary 5.5. Let $R$ be commutative semiartinian ring and $\mathcal{S}$ be a complete irredundant set of noninjective simple modules. Then $\prod_{S \in \mathcal{S}} S$ is poor. Moreover, any submodule $N$ of $\prod_{S \in \mathcal{S}} S$ containing $\oplus_{S \in \mathcal{S}} S$ is poor.

Corollary 5.6. Let $R$ be a commutative ring. Suppose $R$ is Noetherian or semiartinian. Then any poor module has a pauper submodule.

Proof. By Proposition 4.2 and Proposition 5.2.

Theorem 5.7. Let $R$ be a right Artinian ring. Then every noninjective right module contains a pauper module if and only if there is a ring direct sum $R=S \oplus T$, where $S$ is semisimple and $T$ is either zero or has a unique simple right module. In the case $T \neq 0$, $R$ has a unique pauper right module, namely the unique simple submodule of $T$.

Proof. Since $R$ is right Artinian, $R=e_{1} R \oplus e_{2} R \oplus \cdots e_{n} R \oplus f_{1} R \oplus \cdots f_{m} R$, where $e_{1} R, \cdots, e_{n} R$ are the injective pauper right ideals of $R$. Set $S=e_{1} R \oplus e_{2} R \oplus \cdots e_{n} R$ and $T=f_{1} R \oplus \cdots f_{m} R$. Then $\operatorname{Hom}(S, T)=0=\operatorname{Hom}(T, S)$, and so $R=S \oplus T$ is a ring direct sum. If $T=0$ then $R$ is semisimple and the conclusion follows. Suppose $T \neq 0$ and $T$ has two nonisomorphic simple right $T$-modules, say $U, V$. Then $U$ and $V$ both contains pauper submodules by the hypothesis. If the trivial module 0 is pauper, then $T=0$, a contradiction. So both $U$ and $V$ must be pauper modules. Let $A$ be a submodule of $E(U)$ of composition length 2. Then, clearly, $V$ is $A$-injective. But $V$ is poor and $A$ is not semisimple, a contradiction. Therefore $T$ has a unique simple submodule. This proves the necessity.

Sufficiency: If $T=0$, then $R$ is semisimple. So the proof is clear. Suppose $T \neq 0$ and let $U$ be the unique simple right $T$-module. Then $\frac{T}{J(T)} \cong U^{n}$ is poor both as a $T$-module and as a right $R$-module by Proposition 2.2. This implies that $U$ is a poor right $R$-module. Let $M$ be a noninjective right $R$-module. Then $M T \neq 0$, because $M$ is not injective. So that $M$ contains a submodule isomorphic to $U$. This proves the sufficiency. 
We shall prove that any poor module over an Artinian serial ring has a pauper direct summand (i.e. Artinian serial rings satisfy $(\mathrm{U})$ ). We need the following lemma first. Note that, over an Artinian serial ring every module is a direct sum of uniserial modules, and so indecomposable modules are uniserial.

Lemma 5.8. Let $R$ be an Artinian serial ring and $M, N$ indecomposable right $R$-modules. If $\operatorname{cl}(M) \leq \operatorname{cl}(N)$, then $N$ is $M$-injective.

Proof. Let $A$ be a submodule of $M$ and $f: A \rightarrow N$ be any homomorphism. Let $i: N \rightarrow$ $E(N)$ be the inclusion and set $h=i f$. Then there is a homomorphism $g: M \rightarrow E(N)$ such that $\left.g\right|_{A}=h$. Then $\operatorname{cl}(g(M)) \leq \operatorname{cl}(M) \leq \operatorname{cl}(N)$ by the hypothesis. Since $E(N)$ is uniserial, $g(M) \subseteq N$. Therefore $g$ extends $f$. This completes the proof.

Proposition 5.9. Let $R$ be an Artinian Serial ring. Then $S$, the irredundant complete sum of noninjective simple right modules, is a pauper module. Moreover, any poor right module has a direct summand isomorphic to $S$. That is, any poor module has a pauper direct summand. In other words, Artinian Serial rings satisfy $(U)$.

Proof. We know that $S$ is poor by Proposition 2.2. Let us show $S$ is pauper. Suppose the contrary that $S$ is poor but not pauper. Then $S$ has a proper poor direct summand $S^{\prime}$. Then $\operatorname{Hom}\left(U, S^{\prime}\right)=0$ for some noninjective simple right module $U$. Since the ring is Artinian, there is a (nonsemisimple) submodule $Y$ of $E(U)$ such that $\frac{Y}{U}$ is simple. Now, clearly $S^{\prime}$ is $Y$-injective. But $S^{\prime}$ is poor. Contradiction. Therefore $S$ must be pauper.

Let $M$ be a poor module. Then $M=\oplus_{i_{I}} U_{i}$ is a direct sum of uniserial modules $U_{i}$. We shall prove that $M$ has a direct summand isomorphic to $S$, or equivalently for each noninjective simple right module $K$, there is a $t \in I$ such that $K \cong U_{t}$. Suppose the contrary that, there is a noninjective simple right module $T$ such that $T$ is not isomorphic to $U_{i}$ for all $i \in I$. Since $T$ is noninjective, there is an $X \subset E(T)$ such that $\frac{X}{T}$ is simple. In particular, $\operatorname{cl}(X)=2$. Since, for all $i \in I, U_{i}$ is not isomorphic to $T$, for each $U_{j}$ with $\operatorname{Hom}\left(T, U_{j}\right) \neq 0$ we have $\operatorname{cl}(X) \leq \operatorname{cl}\left(U_{j}\right)$. Then $U_{j}$ is $X$-injective by Lemma 5.8. This implies that $M$ is $X$-injective, because $X$ is finitely generated. This contradicts the fact that $M$ is poor. Therefore $M$ has a direct summand isomorphic to $S$. This completes the proof.

\section{Pauper abelian groups}

In this section, we describe pauper abelian groups of torsion-free rank one. Let $p$ be a prime integer. Recall that, for a group $A$ and a nonzero element $a$ of $A$ the $p$-height of $a$, denoted $h_{p}(a)$, is the nonnegative integer $k$ for which $a \in p^{k} A \backslash p^{k+1} A$. If such integer does not exist, then we set $h_{p}(a)=\infty$.

Proposition 6.1. Let $G$ be a pauper abelian group. Then $T(G) \cong \oplus_{p} \mathbb{Z}_{p}$. 
Proof. Let $T_{p}(G)$ be the $p$-primary component of $G$. Since $G$ is poor, $T_{p}(G)$ has a direct summand $V$ isomorphic to $\mathbb{Z}_{p}$. Then $T_{p}(G)=V \oplus H$ for some $H \leq H$. We claim that, $H=0$. Suppose the contrary and let $B$ be the $p$-basic subgroup of $H$. Then $B \neq 0$ and $B$ is a direct sum of cyclic $p$-groups. Let $U$ be a cyclic direct summand of $B$. Now clearly, $U$ is a pure submodule of $G$ and so a direct summand of $G$, because $U$ is bounded. We get $G=U \oplus C$ with $C$ is poor, because $T(C)$ has a direct summand isomorphic to $\oplus_{p} \mathbb{Z}_{p}$. Contradiction. This implies $T_{p}(G)=V$, and so $T(G) \cong \oplus_{p} \mathbb{Z}_{p}$.

Corollary 6.2. A torsion abelian group is pauper if and only if it is isomorphic to $\oplus_{p} \mathbb{Z}_{p}$.

A group $G$ is called a mixed group if it contains both nonzero elements of finite order and elements of infinite order.

Theorem 6.3. [23, Theorem 3.4.] Let a mixed group G satisfy the following conditions:

(a) $\frac{G}{T(G)}$ has rank 1 .

(b) For every prime $p \in P$, the height of all nonzero elements of $T_{p}(G)$ is finite.

(c) Every coset $g+T(G)$ contains an element $x$ such that $h_{p}(x)=h_{p}(g+T(G))$ for all primes $p$.

Then the group $G$ splits.

Lemma 6.4. Let $A$ be a group. Suppose that $T(A)=\oplus_{p} \mathbb{Z}_{p}$ and $\frac{A}{\oplus_{p} \mathbb{Z}_{p}}$ is indecomposable. If $\oplus_{p} \mathbb{Z}_{p}$ is not a direct summand in $A$, then $A$ is pauper.

Proof. $A$ is poor by [2, Theorem 3.1]. Suppose $B$ is a poor direct summand of $A$. Then $B$ contains $\oplus_{p} \mathbb{Z}_{p}$. Then $\frac{B}{\oplus_{p} \mathbb{Z}_{p}}$ is a direct summand of $\frac{A}{\oplus_{p} \mathbb{Z}_{p}}$. Since $\frac{A}{\oplus_{p} \mathbb{Z}_{p}}$ is indecomposable, $B=\oplus_{p} \mathbb{Z}_{p}$ or $B=A$. Therefore $A$ is pauper.

Proposition 6.5. Let $G$ be a pure subgroup of $\prod_{p} \mathbb{Z}_{p}$ containing $\oplus_{p} \mathbb{Z}_{p}$. Then $G$ is pauper.

Proof. Suppose $G$ is pure in $\prod_{p} \mathbb{Z}_{p}$. Then $\frac{G}{\left(\oplus_{p} \mathbb{Z}_{p}\right)}$ is pure in $\frac{\prod_{p} \mathbb{Z}_{p}}{\left(\oplus_{p} \mathbb{Z}_{p}\right)}$. On the other hand, $\frac{\prod_{p} \mathbb{Z}_{p}}{\left(\oplus \mathbb{Z}_{p}\right)}$ is divisible. Therefore $\frac{G}{\left(\oplus \mathbb{Z}_{p}\right)}$ is divisible, because pure subgroups of divisible groups are divisible. So that $\frac{G}{\left(\oplus_{p} \mathbb{Z}_{p}\right)} \cong \mathbb{Q}^{(I)}$ for some index set $I$. In particular, $\operatorname{Rad}\left(\frac{G}{\left(\oplus_{p} \mathbb{Z}_{p}\right)}\right)=$ $\frac{G}{\left(\oplus_{p} \mathbb{Z}_{p}\right)}$. Then $G$ is pauper by Proposition 4.5 .

By Proposition 4.5 the groups $\oplus_{p} \mathbb{Z}_{p}$ and $\prod_{p} \mathbb{Z}_{p}$ are pauper. Moreover, any group $B$ such that $\oplus_{p} \mathbb{Z}_{p} \leq B \leq \prod_{p} \mathbb{Z}_{p}$ is poor by [2, Theorem 3.1]. It is natural to ask which subgroups $A$ of $\prod_{p} \mathbb{Z}_{p}$ containing $\oplus_{p} \mathbb{Z}_{p}$ are pauper. We consider groups $A$ of torsion-free rank 1 . Clearly $R=\frac{A}{\left(\oplus_{p} \mathbb{Z}_{p}\right)}$ is a rational group which can be described by means of the 
characteristic $\chi(r)=\left(h_{2}(r), h_{3}(r), \cdots, h_{p}(r), \cdots\right)$ of any nonzero element $r$ of $R$, (see [12, Chapter 85]).

Theorem 6.6. Let $A$ be a subgroup of torsion-free rank 1 of $\prod_{p} \mathbb{Z}_{p}$ containing $\oplus_{p} \mathbb{Z}_{p}$ and $\chi(r)=\left(h_{2}(r), h_{3}(r), \cdots, h_{p}(r), \cdots\right)$ for some nonzero $r$ in $R=\frac{A}{\oplus_{p} \mathbb{Z}_{p}}$.

(1) If $h_{p}(r)=0$ for only finite number of primes $p$, then $A$ is pauper.

(2) If $h_{p}(r) \neq 0$ for only finite number of primes $p$, then $\oplus_{p} \mathbb{Z}_{p}$ is a direct summand of $A$, so $A$ can not be pauper.

(3) If $h_{p}(r)=0$ for infinite number of primes $p$ and $h_{p}(r) \neq 0$ for infinite number of primes $p$, then $A$ may be or not be pauper.

Proof. Let $P$ be the set of prime integers.

(1) In this case, first let us prove that $\operatorname{Rad}(R) \neq 0$. Let $P^{\prime}$ be the set of primes $p$ such that $h_{p}(r) \neq 0$. Then $r \in p R$ for each $p \in P^{\prime}$, and so $r \in \cap_{p \in P^{\prime}} p R$. By (1), the set $P \backslash P^{\prime}$ is finite, and so $\cap_{p \in P \backslash P^{\prime}} p R \neq 0$. Therefore

$$
\operatorname{Rad} R=\cap_{p \in P} p R=\left(\cap_{p \in P \backslash P^{\prime}} p R\right) \cap\left(\cap_{p \in P^{\prime}} p R\right) \neq 0
$$

because $R$ is of rank 1 . Suppose that there is a nontrivial direct summand $B$ of $A$ that is poor. Then $\oplus_{p \in P} \mathbb{Z}_{p} \leq B$ by Proposition 6.1 , and $A=B \oplus C$ for some nonzero $C \leq A$. Therefore $R=\frac{A}{\left(\oplus_{p} \mathbb{Z}_{p}\right)} \cong \frac{B}{\left(\oplus_{p} \mathbb{Z}_{p}\right)} \oplus C$. Since $R$ is indecomposable and $C \neq 0$, $\frac{B}{\left(\oplus_{p} \mathbb{Z}_{p}\right)}=0$. That is, $B=\oplus_{p} \mathbb{Z}_{p}$ and $C \cong R$. Since $\operatorname{Rad} C \cong \operatorname{Rad} R \neq 0, \operatorname{Rad} A \neq 0$. But $\operatorname{Rad}\left(\prod_{p} \mathbb{Z}_{p}\right)=0$, contradiction. Therefore $A$ is pauper.

(2) By Theorem 6.3, it is sufficient to show that if $h_{q}(r) \neq 0$ for some $q \in P$ and nonzero $r=a+\oplus_{p} \mathbb{Z}_{p} \in R$, then there is $b \in a+\oplus_{p} \mathbb{Z}_{p}$ with $h_{q}(b)=h_{q}(r)$. Let $a=\left(a_{p}\right)_{p \in P} \in A \leq \prod_{p} \mathbb{Z}_{p}$. We claim that $h_{q}(b)=h_{q}(r)$ for $b=\left(b_{p}\right)_{p \in P}$, where

$$
b_{p}= \begin{cases}a_{p}, & \text { if } p \neq q \\ 0, & \text { if } p=q\end{cases}
$$

We will prove that $q^{n} \mid b$ if $h_{q}(r)=n<\infty$, and $q^{n} \mid b$ for every positive integer $n$ if $h_{q}(r)=\infty$. Since $q^{n} \mid r, r=b+\left(\oplus_{p} \mathbb{Z}_{p}\right)=a+\left(\oplus_{p} \mathbb{Z}_{p}\right)=q^{n}\left(c+\oplus_{p} \mathbb{Z}_{p}\right)$ for some $c \in A$. Then $\left(x_{p}\right)_{p \in P}=b-q^{n} c \in \oplus_{p} \mathbb{Z}_{p}$. Clearly $x_{q}=0$. For every $p$ with $x_{p} \neq 0$ there is $y_{p} \in \mathbb{Z}_{p}$ such that $x_{p}=q^{n} y_{p}$ since $p$ and $q$ are relatively prime. Put $y_{p}=0$ if $x_{p}=0$. Then clearly $\left(y_{p}\right)_{p \in P} \in \oplus_{p} \mathbb{Z}_{p}$ and $\left(x_{p}\right)=q^{n}\left(y_{p}\right)$. Since $\oplus \mathbb{Z}_{p} \leq A$, we have $c+\left(y_{p}\right) \in A$ and $b=q^{n}\left(c+\left(y_{p}\right)\right)$, so $q^{n} \mid b$ in $A$.

(3) Let $k=\left(\cdots, k_{p}, \cdots\right)$ be a characteristic such that $k_{p}=0$ for infinite number of $p \in P$ and $k_{p} \neq 0$ for infinite number of $p \in P$. We will give $(i)$ an example of a subgroup $A$ of $\prod_{p} \mathbb{Z}_{p}$ containing $\oplus_{p} \mathbb{Z}_{p}$ as a direct summand with $\chi(r)=k$ for some $r \in \frac{A}{\left(\oplus \mathbb{Z}_{p}\right)}$ and $\left(\right.$ ii) an example of a subgroup $B$ of $\prod_{p} \mathbb{Z}_{p}$ containing $\oplus_{p} \mathbb{Z}_{p}$ which is pauper and $\chi(r)=k$ for some $r \in \frac{A}{\left(\oplus_{p} \mathbb{Z}_{p}\right)}$. 
(i) Define $a=\left(x_{p}\right)_{p \in P} \in \mathbb{Z}_{p}$ by

$$
x_{p}=\left\{\begin{array}{l}
0, \text { if } k_{p} \neq 0 \\
1, \text { if } k_{p}=0
\end{array}\right.
$$

If $0 \neq k_{q}<\infty$ for some $q \in P$, then for each $p \neq q$ there is a unique element $y \in \mathbb{Z}_{p}$ such that $q^{k_{p}} . y_{q}=1$ in $\mathbb{Z}_{p}$ since $q^{k_{p}}$ and $p$ are relatively prime. Put $b(q)=\left(y_{p}\right)_{p \in P \text {. If }}$ $k_{q}=\infty$ for some $q \in P$ then for each $m=1,2, \cdots$ and $p \neq q$ there are unique elements $y_{p_{m}} \in \mathbb{Z}_{p}$ such that $q \cdot y_{p_{1}}=1$ and $q \cdot y_{p_{(m+1)}}=y_{p_{m}}$. Put $b\left(q_{m}\right)=\left(y_{p_{m}}\right)$. Let $C$ be the subgroup of $\prod_{p} \mathbb{Z}_{p}$ generated by all elements $b(q)$ and $b\left(q_{m}\right)$. Then $C \cap\left(\oplus_{p} \mathbb{Z}_{p}\right)=0$ and for $A=C \oplus\left(\oplus_{p} \mathbb{Z}_{p}\right)$ we have $\frac{C \oplus\left(\oplus_{p} \mathbb{Z}_{p}\right)}{\left(\oplus_{p} \mathbb{Z}_{p}\right)} \cong C$. Clearly $\chi(a)=\left(\cdots, k_{p}, \cdots\right)$.

(ii) Since $h_{p}(r) \neq 0$ for infinite number of $p \in P, \frac{R}{r \mathbb{Z}} \cong \oplus_{i \in I} \mathbb{Z}_{p_{i}^{k_{i}}}$, where $I$ is an infinite set and each $k_{i}$ is a positive integer or $k_{i}=\infty$. For the short exact sequence

$$
0 \rightarrow r \mathbb{Z} \rightarrow R \rightarrow \frac{R}{r \mathbb{Z}} \rightarrow 0
$$

we have the following exact sequence

$$
\cdots \rightarrow \operatorname{Hom}\left(r \mathbb{Z}, \oplus \mathbb{Z}_{p}\right) \rightarrow \operatorname{Ext}\left(\frac{R}{r \mathbb{Z}}, \oplus_{p} \mathbb{Z}_{p}\right) \rightarrow \operatorname{Ext}\left(R, \oplus_{p} \mathbb{Z}_{p}\right) \rightarrow \operatorname{Ext}\left(r \mathbb{Z}, \oplus_{p} \mathbb{Z}_{p}\right)=0
$$

Clearly $\operatorname{Hom}\left(r \mathbb{Z}, \oplus_{p} \mathbb{Z}_{p}\right) \cong \oplus_{p} \mathbb{Z}_{p}$ is countable, but

$$
\operatorname{Ext}\left(\frac{R}{r \mathbb{Z}}, \oplus_{p} \mathbb{Z}_{p}\right) \cong \operatorname{Hom}\left(\oplus_{i \in I} \mathbb{Z}_{p_{i}^{k_{i}}}, \oplus_{p} \mathbb{Z}_{p}\right) \cong \prod_{i \in I} \operatorname{Hom}\left(\mathbb{Z}_{p_{i}^{k_{i}}}, \oplus_{p} \mathbb{Z}_{p}\right)
$$

is uncountable.

Therefore $\operatorname{Ext}\left(R, \oplus_{p} \mathbb{Z}_{p}\right) \neq 0$, and so there is a non-splitting (pure) short exact sequence

$$
0 \rightarrow \oplus_{p} \mathbb{Z}_{p} \rightarrow A \rightarrow R \rightarrow 0
$$

Since $\prod_{p} \mathbb{Z}_{p}$ is pure-injective there are homomorphisms $g: A \rightarrow \prod_{p} \mathbb{Z}_{p}$ and $h: R \rightarrow$ $\frac{\prod_{p} \mathbb{Z}_{p}}{\oplus_{p} \mathbb{Z}_{p}}$ such that the diagram

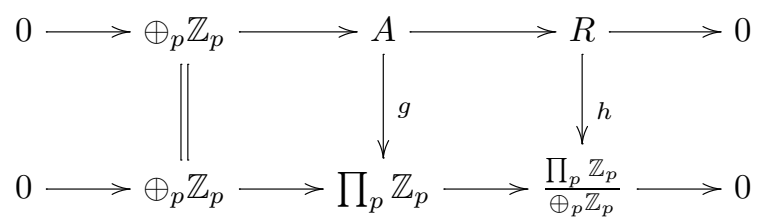

is commutative. If Ker $g \neq 0$, then since the rank of $R$ is 1 and $\frac{\left(\prod_{p} \mathbb{Z}_{p}\right)}{\left(\oplus_{p} \mathbb{Z}_{p}\right)}$ is torsion-free, we have $h=0$. Then $\operatorname{Im} g \subseteq \oplus_{p} \mathbb{Z}_{p}$, that is the first row splits. Contradiction. So $g$ is 
a monomorphism, that is we regard $A$ as a subgroup of $\prod_{p} \mathbb{Z}_{p}$. Now $A$ is pauper by Lemma 6.4.

By [2, Theorem 3.1] the ring of integers satisfies $\left(\mathrm{U}^{*}\right)$. On the other hand, the class of torsion groups satisfies (U) by $[2$, Corollary 3.2]. It is natural to ponder whether $\mathbb{Z}$ satisfies (U) and to try to extend the result to other classes of abelian groups. The following result is a step in that direction.

Corollary 6.7. If an Abelian group A satisfies the conditions in Theorem 6.6 and is not pauper then $A=\left(\oplus_{p} \mathbb{Z}_{p}\right) \oplus C$ for some nonzero submodule $C$ of $A$. It follows that, the class of torsion-free rank one groups satisfies $(U)$.

Proof. Suppose $A$ is not pauper. Then there is a proper and poor submodule $B$ of $A$ such that $A=B \oplus C$. Since $B$ is poor, $\oplus_{p} \mathbb{Z}_{p} \leq B$. Note that, $C \cap\left(\oplus_{p} \mathbb{Z}_{p}\right)=0$. If $\oplus_{p} \mathbb{Z}_{p}$ is properly contained in $B$, then $\frac{A}{\oplus_{p} \mathbb{Z}_{p}} \cong \frac{B}{\oplus_{p} \mathbb{Z}_{p}} \oplus C$ is a direct sum two nonzero groups. But $\frac{A}{\oplus_{p} \mathbb{Z}_{p}}$ is indecomposable. Therefore we must have $B=\oplus_{p} \mathbb{Z}_{p}$, and this completes the proof.

\section{Questions}

We would like to close by highlighting what we consider the most pressing questions we have left unanswered at this time.

Q1. Characterize pauper abelian groups.

Q2. Determine the structure of rings that satisfy $(U)$ ? In particular, does the ring of integers satisfy $(U)$ ?

Q3. To determine the structure of poor modules over arbitrary commutative Noetherian rings.

Q4. Determine whether (commutative) Noetherian rings satisfy $\left(\mathrm{U}^{*}\right)$.

\section{References}

[1] A.N. Alahmadi, M. Alkan, S. López-Permouth, Poor modules: the opposite of injectivity, Glasg. Math. J. 52 (A) (2010) 7-17.

[2] R. Alizade, E. Büyükaşık, Poor and pi-poor abelian groups, Comm. Algebra 45 (1) (2017) $420-427$.

[3] R. Alizade, E. Mermut, Proper classes related with complements and supplements, Palest. J. Math. 4 (Spec. 1) (2015) 471-489.

[4] R. Alizade, D. Dede-Sipahi, Modules and abelian groups with minimal (pure-) projectivity domains, J. Algebra Appl. 16 (11) (2017).

[5] F.A. Anderson, K.R. Fuller, Rings and Categories of Modules, 2nd ed., Graduate Texts in Mathematics, vol. 13, Springer-Verlag, New York, 1992.

[6] P. Aydoğdu, B. Saraç, On Artinian rings with restricted class of injectivity domains, J. Algebra 377 (2013) 49-65.

[7] E. Büyükaşık, E. Enochs, J.R. García Rozas, G. Kafkas-Demirci, S. López-Permouth, L. Oyonarte, Rugged modules: the opposite of flatness, Comm. Algebra 46 (2) (2018) 764-779.

[8] N.V. Dung, P.F. Smith, On semi-Artinian V-modules, J. Pure Appl. Algebra 82 (1) (1992) $27-37$. 
[9] N. Er, S. López-Permouth, N. Sökmez, Rings whose modules have maximal or minimal injectivity domains, J. Algebra 330 (2011) 404-417.

[10] N. Er, S. López-Permouth, N.K. Tung, Rings whose cyclic modules have restricted injectivity domains, J. Algebra 466 (2016) 208-228.

[11] A. Facchini, Module Theory, Birkhauser, 1998.

[12] L. Fuchs, Infinite Abelian Groups, vol. II, Pure and Applied Mathematics, vol. 36-II, Academic Press, New York-London, 1973, MR0349869.

[13] L. Fuchs, L. Salce, Modules over Non-Noetherian Domains, Mathematical Surveys and Monographs, vol. 84, AMS, 2001.

[14] K.R. Goodearl, Singular Torsion and the Splitting Properties, Memoirs of the American Mathematical Society, vol. 124, AMS, 1972.

[15] T.Y. Lam, Lectures on Modules and Rings, Graduate Texts in Mathematics, vol. 189, SpringerVerlag, New York, 1999.

[16] S.R. López-Permouth, J.E. Simental, Characterizing rings in terms of the extent of the extent of the injectivity and projectivity of their modules, J. Algebra 362 (1) (2012) 56-69.

[17] E. Matlis, Modules with descending chain conditions, Trans. Amer. Math. Soc. 97 (1960) 495-508.

[18] G.O. Michler, O.E. Villamayor, On rings whose simple modules are injective, J. Algebra 25 (1973) 185-201.

[19] S.H. Mohamed, B.J. Müller, Continuous and Discrete Modules, London Mathematical Society Lecture Note Series, vol. 147, Cambridge University Press, Cambridge, 1990.

[20] J.J. Rotman, An Introduction to Homological Algebra, 2nd ed., Universitext, Springer, New York, 2009, MR2455920.

[21] W. Scott, Group Theory, Dover, 1964, 1987.

[22] R. Ware, Endomorphism rings of projective modules, Trans. Amer. Math. Soc. 155 (1971) $233-256$.

[23] V.S. Zuravskiy, On splitting of some mixed Abelian groups, Math. Sb. 48(90)-4 (1959) $499-508$. 